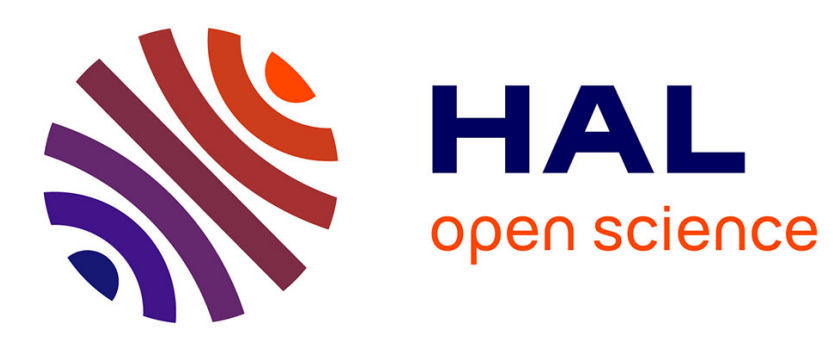

\title{
Resilience-Based Component Importance Measures for Critical Infrastructure Network Systems
}

\author{
Yi-Ping Fang, Nicola Pedroni, Enrico Zio
}

\section{To cite this version:}

Yi-Ping Fang, Nicola Pedroni, Enrico Zio. Resilience-Based Component Importance Measures for Critical Infrastructure Network Systems. IEEE Transactions on Reliability, 2016, 65 (2), pp.502-512. 10.1109/TR.2016.2521761. hal-01436576v2

\section{HAL Id: hal-01436576 \\ https://hal-centralesupelec.archives-ouvertes.fr/hal-01436576v2}

Submitted on 16 Feb 2016

HAL is a multi-disciplinary open access archive for the deposit and dissemination of scientific research documents, whether they are published or not. The documents may come from teaching and research institutions in France or abroad, or from public or private research centers.
L'archive ouverte pluridisciplinaire HAL, est destinée au dépôt et à la diffusion de documents scientifiques de niveau recherche, publiés ou non, émanant des établissements d'enseignement et de recherche français ou étrangers, des laboratoires publics ou privés. 


\title{
Resilience-Based Component Importance Measures for Critical Infrastructure Network Systems
}

\author{
Yi-Ping Fang, Nicola Pedroni, and Enrico Zio, Senior Member, IEEE
}

\begin{abstract}
In this paper, we propose two metrics, i.e., the optimal repair time and the resilience reduction worth, to measure the criticality of the components of a network system from the perspective of their contribution to system resilience. Specifically, the two metrics quantify: 1) the priority with which a failed component should be repaired and re-installed into the network and 2) the potential loss in the optimal system resilience due to a time delay in the recovery of a failed component, respectively. Given the stochastic nature of disruptive events on infrastructure networks, a Monte Carlo-based method is proposed to generate probability distributions of the two metrics for all of the components of the network; then, a stochastic ranking approach based on the Copeland's pairwise aggregation is used to rank components importance. Numerical results are obtained for the IEEE 30-bus test network and a comparison is made with three classical centrality measures.
\end{abstract}

Index Terms-Component importance measures, critical infrastructure, stochastic ranking, system recovery, system resilience.

\section{ACRONYMS AND ABBREVIATIONS}

CI Critical infrastructure.

CIP Critical infrastructure protection.

WCDR World conference on disaster reduction.

CIM Component importance measure.

MIP Mixed integer programming.

CM Copeland's method.

CDF Cumulative distribution function.

ORT Optimal repair time.

RRW Resilience reduction worth.

Manuscript received November 03, 2014; revised April 28, 2015; accepted January 20, 2016. Associate editor: S. Shieh.

Y.-P. Fang was with the Chair on Systems Science and the Energetic Challenge, École Centrale Paris and Supélec (CentraleSupélec), 92295 ChâtenayMalabry, France. He is now with the Department of Mechanical and Process Engineering, ETH Zürich 8092, Switzerland (e-mail: fangy@ethz.ch).

N. Pedroni is now with the Chair on Systems Science and the Energetic Challenge, École Centrale Paris and Supélec (CentraleSupélec), 92295 ChâtenayMalabry, France (e-mail: nicola.pedroni@ecp.fr).

E. Zio is with the Chair on Systems Science and the Energetic Challenge, 92295 Châtenay-Malabry, France, École Centrale Paris and Supélec (CentraleSupélec), Paris, France, and also with the Department of Energy, Politecnico di Milano, Milan 20133, Italy (e-mail: enrico.zio@ecp.fr; enrico.zio@supelec.fr; enrico.zio@polimi.it).

Color versions of one or more of the figures in this paper are available online at http://ieeexplore.ieee.org.

Digital Object Identifier 10.1109/TR.2016.2521761

\section{NOTATION}

$F(t) \quad$ System performance function

$\widehat{F}(t) \quad$ Target system performance function.

$R(t) \quad$ Time-dependent system resilience.

$V \quad$ Set of nodes.

E Set of arcs

$E^{\prime} \quad$ Set of disrupted arcs.

$G(V, E) \quad$ Network comprising a set of nodes $V$ connected by a set of arcs $E$.

$V_{S}, V_{T}, V_{D} \quad$ Supply, transship, and demand nodes, respectively.

$P(i j) \quad$ Capacity of link $i j \in E$.

$P_{i}^{s} \quad$ Supply capacity of node $i \in V_{S}$.

$P_{j}^{D} \quad$ Demand of node $j \in V_{D}$.

$T \quad$ Total recovery time.

$f_{j}(t) \quad$ Flow received by demand node $j$ at time $t$.

$f_{i j}(t) \quad$ Flow from node $i$ to $j$ through arc $i j$ at time $t$.

$s_{i j}(t) \quad$ State variable of arc $i j$ at time $t$.

$p_{e}(i j) \quad$ Failure probability of arc $i j$ under event $e$.

$T_{i j}^{\text {opt }} \quad$ Optimal repair time of arc $i j$.

$R R W_{i j}\left(\Delta t_{0}\right)$ Resilience reduction worth of arc $i j$ under delay $\Delta t_{0}$.

$C(i j) \quad$ Copeland score of arc $i j$.

$C_{i j}^{B}$

$C_{i j}^{F}$

$C_{i j}^{R W}$

Shortest path betweenness centrality.

Flow betweenness centrality.

Random walk betweenness centrality.

\section{INTRODUCTION}

O OMPLEXITY of critical infrastructures (CIs), such as power grids, the Internet, transportation networks, and so forth, is increasing. Disruptive events, whether they are malevolent attacks, natural disasters, or human-caused accidents, can have significant impacts on these real-world complex networks composed of numerous interconnected functional and structural elements. 
Justifiably, then, critical infrastructure protection (CIP) has become a priority for all nations [1]. The focus has been traditionally placed on physical protection and asset hardening [2]-[5]. However, in recent years, lessons learned from some catastrophic accidents have pushed part of the focus on the concept of "resilience" [6], [7]. The outcomes of the 2005 World Conference on Disaster Reduction (WCDR) witness the significance of introducing the term "resilience" into the disaster discourse, giving birth to a new culture of disaster response [8]. Consequently, government policy has also evolved to encourage efforts that would allow assets to continue operating at some level or quickly return to full operation after the occurrence of disruptive events [9].

"Resilience" comes from the Latin word "resilio" that literally means "to leap back" and denotes a system attribute characterized by the ability to recover from challenges or disruptive events. The Merriam-Webster dictionary defines resilience as "the ability to recover from or adjust easily to misfortune or change." In this view, systems should not only be reliable, i.e., having an acceptably low failure probability, but also resilient, i.e., having the ability to optimally recover from disruptions of the nominal operating conditions [10], [11].

In this context, the present paper addresses the issue of quantifying the importance of components in contributing to the resilience of a critical infrastructure. Component importance measures (CIMs) have been thoroughly studied in the field of reliability theory and risk analysis. Various analytical and empirical CIMs have been proposed in the literature, e.g., Birnbaum [12], Fussell-Vesely [13], Reliability Achievement/Reduction Worth [14], [15], Barlow-Proschan [45], Natvig [46], and their extensions [16]-[20], [34], [47], [48]. CIMs have been shown valuable in establishing direction and prioritization of actions related to an upgrading effort (e.g., reliability improvement) in system design, or in suggesting the most efficient way to operate and maintain system status. However, none of the existing classical CIMs based on the reliability concept are directly applicable to the post-disaster phase, since there is no scope to exhibit reliability after the occurrence of system failure.

The role that a component plays in a network system has been measured by various so-called centrality measures, looking from the point of view of the complex interaction and communication flow in the network [21], [22]. Classical topological centrality measures are the degree centrality [23], [24], the closeness centrality [24]-[26], the betweenness centrality [24], and the information centrality [27]. They specifically rely on topological information to qualify the importance of a network component. Additionally, Freeman et al. [28] proposed a flow betweenness centrality measure based on the idea of maximum network flow; Newman [29] suggested a random walk betweenness measure that counts essentially all paths between vertices and which makes no assumptions of optimality; Jenelius et al. [30] proposed several vulnerability-based importance measures for transportation networks; Hines and Blumsack [31] introduced an "electrical centrality" measure for electrical networks by taking into account the electrical topology of the network; Zio and Piccinelli [32] provided a randomized flow model-based centrality measure specifically for electrical networks; Zio and Sansavini [33] introduced component criticality measures from the cascade failure process point of view, for general network systems. Nevertheless, none of these analyses takes into account the dynamics of system recovery from the effects of a disruptive event.

Resilience-based metrics of component criticality with respect to their influence on the overall resilience of the system (i.e., on the system's ability to quickly recover from a disruptive event) can be helpful for preparing an efficient component repair checklist in the event of system failure [34]. Natvig et al. introduced a dual extended Natvig measure for repairable systems: according to this measure, the components that are considered important are those whose repair reduces significantly the expected time of residence of the system in the worst states [34]. Hence, this dual Natvig measure is a resilience measure for multistate components in a multistate system. A dual extension of the Barlow-Proschan measure has also been suggested for multistate repairable systems, based on the probability that the repair of the $i$ th component is the cause of a system state improvement, given that this has occurred [48].

Recently, Barker et al. [35] introduced two resilience-based network component importance metrics. Although the resilience definition, which the importance metrics rely on, actually embraces the temporal dimension of system recovery, it can be considered "memoryless": in the sense that the system resilience metric $R_{F}\left(t_{r} \mid e_{j}\right)$ at a given time $t_{r}$ does not take into account the information about the restoration behavior before time $t_{r}$. Thus, it may happen that different restoration curves with different levels of favorability have the same value of resilience $R_{F}\left(t_{r} \mid e_{j}\right)$. In addition, the two metrics introduced in [35] seek to quantify the effect that the disruption, rather than the recovery (behavior), of an individual component has on the system resilience, implying that the resilience improvement is achieved by actions related to system protection and fortification efforts in system design, although they have also been applied to compare network recovery strategies [36].

In this study, a new definition of system resilience and a resilience optimization framework are first presented, based on which we then introduce two network components importance measures, namely, the optimal repair time and the resilience reduction worth, useful for prioritizing restoration activities. The two measures quantify: 1) the priority with which a failed component should be repaired and re-installed into the network and 2) the potential loss in the optimal system resilience due to a time delay in the recovery of a failed component, respectively. A stochastic ranking technique proposed by Barker et al. [35], which is based on the Copeland's pairwise aggregation [37], is applied in this paper to rank the components' criticalities.

As a case study, the IEEE 30-bus test network is considered: the criticalities of the components computed by the proposed indicators are compared with those produced by three classical measures of betweenness centrality [28], [29], [38].

It is noted that the differences of the resilience-based CIMs proposed in the present paper with respect to that in [35] mainly fall into the following two aspects: 1) the concept and definition of system resilience which the CIMs rely on takes into account the cumulative restored system functionality and 2) the focus of the proposed resilience-based CIMs is to quantify the effect that the recovery, rather than the disruption, of individual 


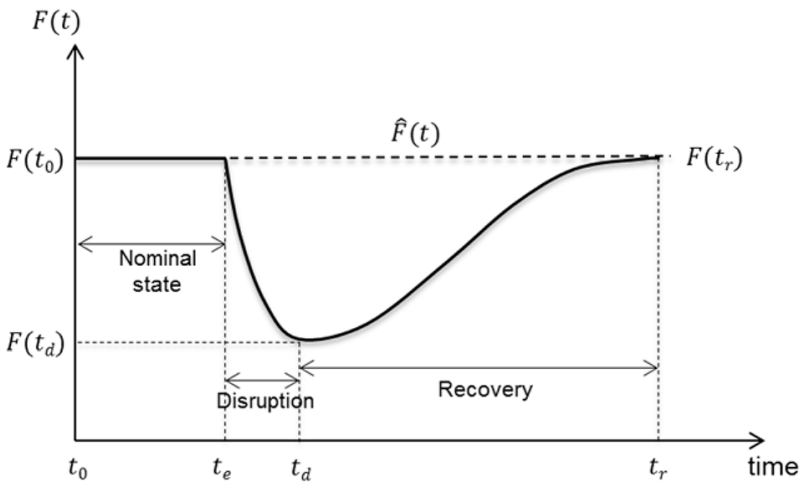

Fig. 1. Generic system performance transition curve under the occurrence of disruption.

components has on the global system resilience, for most effective system (recovery) operation.

The remainder of this paper is organized as follows. Section II provides the general framework of the study, recalling the definition of system resilience and the resilience optimization model. In Section III, two measures of component criticality for system resilience, and a simulation methodology for their calculation and ordering are presented. Section IV illustrates the calculation of the proposed metrics on the IEEE 30-bus test network: the obtained components rankings are compared with those produced by classical betweenness centrality measures. Concluding remarks are drawn in Section V.

\section{Methodological Background: System Resilience DEFINITION AND OPTIMIZATION FOR INFRASTRUCTURE NETWORK SYSTEMS}

This section provides the definition of system resilience and the resilience optimization framework, which serve as methodological background for the resilience-based component importance measures that will be discussed in Section III.

\section{A. System Resilience Definition}

As illustrated in Fig. 1, a quantifiable and time-dependent system performance function (also called system level delivery function or figure-of-merit) $F(t)$ is the basis for the assessment of system resilience [8], [35], [36]. It has a nominal value $F\left(t_{0}\right)$ under nominal operating conditions. The system operates at this level until suffering a disruptive event at time $t_{e}$. The disruption generally deteriorates system performance to some level $F\left(t_{d}\right)$ at time $t_{d}$. Then, recovery action is started, affecting and improving system performance until it achieves, at a later time $t_{r}$, a targeted level of performance $F\left(t_{r}\right)$ that could be the same, close to, or better than original system performance $F\left(t_{0}\right)$, for which recovery is considered completed. The dotted curve $\widehat{F}(t)$ in Fig. 1 denotes the targeted system performance if not affected by disruption, which is generally evolving due to the dynamic nature of service demand and system upgrading; in this study, it is assumed to be equal to $F\left(t_{0}\right)$ and remain invariant, for simplicity of illustration. In addition, it is noted that various strategies exist for recovery activities, and system performance is ultimately a function of recovery decisions. The period of $t_{d} \leq t \leq t_{r}$ is generally considered as the recovery time [8].

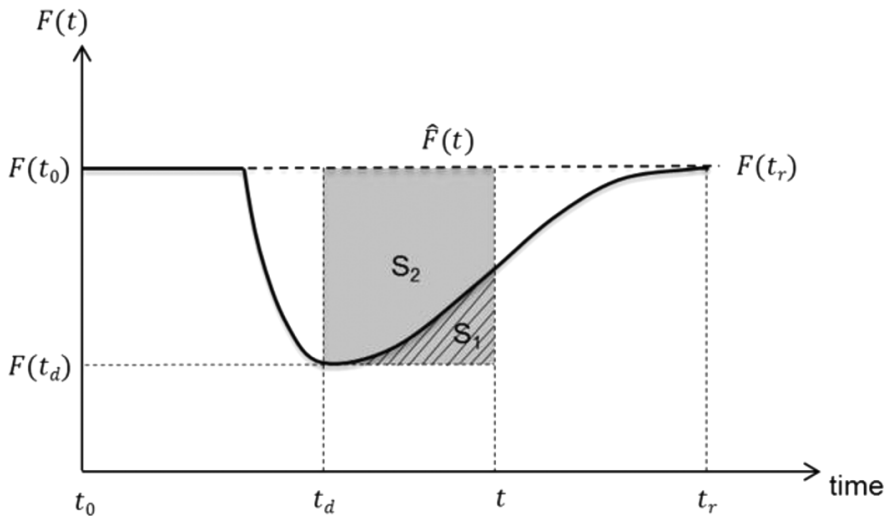

Fig. 2. Conceptual illustration of the proposed resilience measurement.

Let $R(t)$ be the resilience of a system at time $t\left(t \geq t_{d}\right)$. In its basic form, $R(t)$ describes the cumulative system functionality that has been restored at time $t$, normalized by the expected cumulative system functionality supposing that the system has not been affected by disruption during this time period and given as

$$
R(t)=\frac{\int_{t_{d}}^{t}\left[F(\tau)-F\left(t_{d}\right)\right] d \tau}{\int_{t_{d}}^{t}\left[\widehat{F}(\tau)-F\left(t_{d}\right)\right] d \tau}, t \geq t_{d} .
$$

Graphically, $R(t)$ is quantified by the ratio of the area with diagonal stripes $S_{1}$ to the area of the shaded part $S_{2}$, as shown in Fig. 2.

Note that the formulation in (1) focuses mainly on the recoverability dimension of resilience and $R(t)$ is in the range of [0, 1]. $R(t)=0$ when $F(t)=F\left(t_{d}\right)$, which means that a system has not recovered from its disrupted state (i.e., there has been no "resilience" action); $R(t)=1$ when $F(t)=\widehat{F}(t)$, which corresponds to the ideal case where a system recovers to its target state immediately after disruption. This resilience quantification is consistent with the original meaning of the concept of resilience and is capable of measuring at the same time the magnitude and rapidity of system recovery action. More importantly, this definition of system resilience is not memoryless since it considers cumulatively the system functionality restored, differently from [35].

\section{B. System Resilience Optimization}

A disruptive event could impact one or more components of an infrastructure network system. In the case of multiple component failures, a systemic recovery action should be undertaken with the order of failed components to repair such that system resilience is maximal, i.e., to achieve optimal (restored) cumulative system functionality over the recovery time considered.

A variety of frameworks of optimization for post-disaster recovery of an infrastructure network system can be designed, focusing on different aspects of the restoration strategy, e.g., the order of repair of the damaged components, where and how to allocate repair resources and so forth. This study focuses on the significance of the recovery of a component with respect to the resilience of the system. Consequently, the optimization is designed to find the optimal order of repair of the set of failed 
components with the objective of achieving maximum system resilience over the restoration time horizon.

The mathematical model for the resilience optimization concerns a network $G(V, E)$ comprising a set of nodes $V$ connected by a set of links or arcs $E$. The network nodes are distinguished in supply nodes $V_{S}$, transshipment nodes $V_{T}\left(V_{D}\right)$, and demand nodes $V_{S} \cup^{V_{T}} \cup^{V_{D}}=V$. Each arc $i j \in V$ has an associated capacity $P(i j) \in R_{0}^{+}$, each supply node $i \in V_{S}$ has a supply capacity per time unit $P_{i}^{s} \in R_{0}^{+}$and each demand node $j \in V_{D}$ has a demand $P_{j}^{D} \in R_{0}^{+}$per time unit. Network flow is delivered from supply nodes to demand nodes respecting the flow capacities of the links and supply/demand capacities of the nodes. The performance of the network is evaluated by determining the maximum amount of flow that can be received by the demand nodes. Formally, the system performance function is defined as

$$
F(t)=\sum_{j \in V_{D}} f_{j}(t)
$$

where $f_{j}(t)$ represents the amount of flow received by demand node $j$ at time $t$.

Disruptions happen and create damages to nodes and/or links in the network, which is modeled as removal of a subset of arcs, $E^{\prime} \subset E$, from the network. ${ }^{1}$ The arcs in set $E^{\prime}$ are viewed as non-operational immediately after the disruption. System performance $F(t)$ achieves its minimum value at this time, which is seen as the initial stage of system restoration that we focus on (we set $t=0$ at this time for computational convenience, i.e., $\left.F_{\min }=F(0)\right)$.

The recovery optimization framework aims at identifying the subset of links in $E^{\prime}$ to repair and the order, in which the links should be repaired so as to achieve maximum system resilience over the restoration horizon $T \in Z^{+}$. In this study, link repairs are assumed to be discrete tasks and only a single arc can be repaired at a given time period. Thus, discrete time periods $t=1, \ldots, T$ are considered, hereafter, instead of the continuous one in the resilience definition of (1). In addition, we do not model in detail the mechanisms and procedures according to which a single failed component is repaired; rather, the focus is on the identification of the exact (optimal) time when the disrupted arcs should be brought back online and, thus, on the temporal sequence of the restoration actions on all the failed components. Obviously, the inclusion of possibly different repair times for different components may produce a different component ranking. However, this would be automatically accounted for in the procedure by the inclusion of proper "hard" constraints and, thus, it would not impair the applicability and generality of the approach . By combining (1) and (2), system resilience to be maximized at time $T$ is given by

$$
R(T)=\frac{\sum_{t=1}^{t=T}\left[\sum_{j \in V_{D}} f_{j}(t)-F_{\min }\right]}{T \cdot\left(\sum_{j \in V_{D}} P_{j}^{D}-F_{\min }\right)}
$$

${ }^{1}$ For nodes, they can be converted to equivalent arcs by introducing additional arcs and nodes into the network, i.e., by "splitting" a node into two nodes and an arc. where $\sum_{j \in V_{D}} P_{j}^{D}=\widehat{F}(t)$ is the target system performance. The variables of the resilience optimization problem include: 1) continuous variables $f_{i j}(t) \in R_{0}^{+}, i j \in E$ and $t=1, \ldots, T$, that denote the flow moving from node $i$ to node $j$ through link $i j$ at time unit $t$; 2) continuous variables $f_{j}(t) \in R_{0}^{+}, j \in V_{D}$, that represent the amount of flow received by demand node $j$ at time unit $t$, and 3) binary state variables $s_{i j}(t), i j \in E$ and $t=1, \ldots, T$, such that $s_{i j}(t)=1$ if arc $i j$ is operational and $s_{i j}(t)=0$ if arc $i j$ is not operational at time unit $t$. We are interested in optimizing the resilience over the whole restoration process: thus, the timespan $T$ is the total recovery time, defined as the period necessary to restore the system functionality to the same level as the original system. Consequently, the formulation of the resilience optimization problem is as follows:

$$
\max \frac{\sum_{t=1}^{t=T}\left[\sum_{j \in V_{D}} f_{j}(t)-F_{\min }\right]}{T \cdot\left(\sum_{j \in V_{D}} P_{j}^{D}-F_{\min }\right)}
$$

subject to

$$
\begin{gathered}
\sum_{(i, j) \in E} f_{i j}(t)-\sum_{(j, i) \in E} f_{j i}(t) \leq P_{i}^{s}, \forall i \in V_{S}, \forall t \\
\sum_{(i, j) \in E} f_{i j}(t)-\sum_{(j, i) \in E} f_{j i}(t)=0, \forall i \in V_{T}, \forall t \\
\sum_{(i, j) \in E} f_{i j}(t)-\sum_{(j, i) \in E} f_{j i}(t)=-f_{j}(t), \forall i \in V_{D}, \forall t \\
0 \leq f_{j}(t) \leq P_{j}^{D}, \forall i \in V_{D}, \forall t \\
\quad f_{i j}(t) \leq s_{i j}(t) P(i j), \forall i j \in E, \forall t \\
\sum_{i j}(t) \leq s_{i j}(t+1), \forall i j \in E, \forall t \\
\quad\left[s_{i j}(t)-s_{i j}(t-1)\right]=1, \forall t \\
s_{i j}(t) \in\{0,1\}, s_{i j}(0)=0, \forall i j \in E^{\prime}, \forall t
\end{gathered}
$$

The objective (4) is to maximize the system resilience over the time horizon of recovery. Constraints (5)-(9) are typical network flow constraints over the links and supply/demand nodes in the network in period $t$. They ensure that: the flow generated at a supply node does not exceed its supply capacity (5); the amount of net injected flow at a transshipment node is zero (6); the amount of net injected flow at a demand node is equal to the received flow at the node (7) while not exceeding its requested demand (8); the flow on an operational link does not exceed its capacity and there is no flow passing through an arc if the arc is failed (9). Constraint (10) ensures that once an arc has been restored at time $t$, it will keep operational thereafter. Finally, constraint (11) ensures that only a single arc can be repaired at any given timeslot.

This resilience optimization above defined is a typical mixed integer programming (MIP) problem. A commercial optimization solver Cplex [39] is used in this study for its solution. It is noted that this resilience optimization model is only applied for the purpose of illustration of resilience-based component importance metrics. More complex optimization models (e.g., taking into account the cost and duration of repairing a particular failed 
link) can be adopted in other application cases. Besides, a specific application involving the 400-kV French Power Transmission Network of the proposed resilience (restoration) optimization problem has been presented in [49]. While the present study mainly focuses on the definition of resilience-based CIMs and the analysis of their properties, as mentioned before.

\section{MAth ResilienCE-BASED CIMS FOR INFRASTRUCtURE NETWORK SYSTEMS}

\section{A. CIM Definition}

As described in Section II-B, the analysis concerns a network $G(V, E)$ comprising a set of nodes $V$ and a set of links $E$. The binary state variable of arc $i j$ at time $t$ is defined by $s_{i j}(t)$, $\forall i j \in E$. The initial impact experienced by the network after a disruptive event $e$ at time $t=0$ is represented by the removal of a subset of arcs $E^{\prime} \subset E$ from the network, setting $s_{i j}(0)=0$, $\forall i j \in E^{\prime}$. We introduce the failure probability of arc $i j$ under event $e, p_{e}(i j)$

$$
P\left[s_{i j}(0)=0 \mid e\right]=p_{e}(i j), \forall i j \in E .
$$

Equation (13) describes how individual components (links) are initially affected by a disruptive event $e$. Section II-B explains how these failed components optimally recover from the disruption state following the event. Finally, (1) incorporates these dimensions to quantify system resilience.

When considering component criticality in a resilience setting, we are interested in understanding: 1) the optimal time to repair the failed components in order to maximize system resilience and 2) the effect that the timely recovery of the components have on the overall resilience of the system. These concepts are at the basis of the definition of the two resilience-based importance measures here proposed.

Given a particular initial failure state, the optimal repair time $T_{i j}^{\mathrm{opt}}$ of a failed arc $i j$ is defined as the time when the arc $i j$ is restored to operating status so to maximize the system resilience over the recovery time $T$, given by

$$
T_{i j}^{\text {opt }}=\arg \max _{T_{i j} \in[0, T]} R(T) .
$$

It can be obtained by first solving the MIP problem (4)-(12) and then computing it based on the state variables of the arc $i j$ as

$$
T_{i j}^{\mathrm{opt}}=\sum_{t=0}^{T}\left(1-s_{i j}(t)\right) .
$$

The timespan for restoration $T$ is chosen as the time period necessary to restore the system functionality to the same level as the original system. It is noted that the optimal repair time $T_{i j}^{\text {opt }}$ offers an explicit quantification of the priority that should be given to the reparation and installation of arc $i j$ into the network. Low values of $T_{i j}^{\mathrm{opt}}$ indicate higher priority of being repaired and re-installed into the network, i.e., higher ranking of the component in the repair checklist.

To account for the delay in the restoration of a particular link $i j$, a resilience reduction worth (RRW) metric is introduced as

$$
R R W_{i j}\left(\Delta t_{0}\right)=\frac{R^{\mathrm{opt}}(T)-R^{\mathrm{opt}}\left(T \mid T_{i j} \geq T_{i j}^{\mathrm{opt}}+\Delta t_{0}\right)}{R^{\mathrm{opt}}(T)}
$$

where $R^{\text {opt }}(T)$ represents the optimal system resilience at restoration time $T, R^{\mathrm{opt}}\left(T \mid T_{i j} \geq T_{i j}^{\mathrm{opt}}+\Delta t_{0}\right)$ corresponds to the optimal system resilience at time $T$ if link $i j$ cannot be repaired until time $\left(T_{i j}^{\mathrm{opt}}+\Delta t_{0}\right)$, where $\Delta t_{0}$ is the delay with respect to its optimal repair time $T_{i j}^{\text {opt }}$ given by (14). Equation (16) quantifies the potential (normalized) loss in optimal system resilience due to a delay $\Delta t_{0}$ in the repair of link $i j$. This metric is comparable to the so-called reliability reduction worth [40], which measures the potential damage caused to the system reliability by the failure of a particular component. It can provide valuable information to guide the recovery process of a particular component. Components with high values of $R R W_{i j}\left(\Delta t_{0}\right)$ should be given high priority in the restoration process, e.g., be assigned adequate restoration resources to avoid delays that would have a more significant impact on system restoration.

It is noted that the resilience-based CIMs in [35] are introduced to quantify the effect that the disruption, rather than the recovery (behavior), of an individual component has on the system resilience (represented by the system recovery time). Specifically, the first metric in [35] measures the contribution that the disruption of link $i j$ has on the system recovery time, weighted by the ratio of system service loss due to the disruption effect on link $i j$ to the maximum loss among all of the links. The second metric in [35] quantifies how the system recovery time is improved if link $i j$ is invulnerable. Both definitions imply that the resilience improvement is achieved by actions related to system protection and fortification efforts in system design. However, the two resilience-metrics introduced in the present paper measure the effect that the recovery of an individual component has on the global system resilience, by quantifying: 1) the priority with which a failed component should be repaired and re-installed into the network and 2) the potential loss in the optimal system resilience due to a time delay in the recovery of a failed component, respectively. Hence, our resilience-based metrics are able to suggest the most effective way for system operation, i.e., to help the implementation of: 1) recovery schedule planning and 2) restoration resources allocation.

\section{B. Methodology for Component Importance Ordering}

Ordering network links recovery on the basis of the values of the criticality measures described above, i.e., the optimal repair time $T_{i j}^{\mathrm{opt}}$ and resilience reduction worth $R R W_{i j}$ (fixed $\Delta t_{0}$ ), requires quantifying the effect of timely repairing these links on the overall resilience of the system. Given the stochastic nature of disruptive events in terms of components failures after the event, the resilience-based criticality measures introduced are not represented by deterministic values, but rather by probability distributions. Therefore, given a network $G(V, E)$ under a disruptive event $e$, we first apply a Monte Carlo-based method to generate distributions of optimal repair time $T_{i j}^{\mathrm{opt}}$ and resilience reduction worth $R R W_{i j}\left(\Delta t_{0}\right)$ for all of the links in the network. Then, we rank links importance using a Copeland's pairwise aggregation-based stochastic approach proposed by Barker et al. [35]. The detailed steps of the algorithm are as follows.

1) A network $G(V, E)$ is initially operating with a given parameters setting: flow demand $P_{j}^{D}$ of all of the demand nodes in $V_{D}$, supply capacity $P_{i}^{S}$ of all of the supply nodes in $V_{S}$ and link capacity $P(i j)$ for all of the network $\operatorname{arcs}$ in $E$. 
2) A failure configuration of the network is randomly sampled on the basis of the failure probabilities of each arc in the system given by (13), under a disruptive event $e$ at initial time $t=0$. The operation state variables of failed links are set to 0 , i.e., $s_{i j}(0)=0, \forall i j \in E^{\prime}$.

3) The resilience optimization model of (4)-(12) is applied and solved by Cplex to obtain the optimal strategy of network recovery, i.e., the optimal repair time $T_{i j}^{\text {opt }}$ for each failed arc $i j \in E^{\prime}$.

4) In order to evaluate the second importance measure $R R W_{i j}\left(\Delta t_{0}\right)$, for each failed arc $i j \in E^{\prime}$, the additional constraint that the restoration of arc $i j$ should not be accomplished earlier than $T_{i j}^{\text {opt }}+\Delta t_{0}$ (i.e., $\left.T_{i j} \geq T_{i j}^{\mathrm{opt}}+\Delta t_{0}\right)$ is added to the optimization model of (4)-(12). Then, $R^{\mathrm{opt}}\left(T \mid T_{i j} \geq T_{i j}^{\mathrm{opt}}+\Delta t_{0}\right)$ is obtained by solving this "modified" optimization model by Cplex. Finally, the resilience reduction worth $R R W_{i j}\left(\Delta t_{0}\right)$ for each arc $i j$ is recorded.

5) To account for the stochasticity of the disruptive event in terms of arcs failures, repeat Step 2 to Step 4 for a chosen number $\aleph$ of iterations, generating probability distributions for $T_{i j}^{\mathrm{opt}}$ and $R R W_{i j}\left(\Delta t_{0}\right)$, for all of the links in the network.

6) Given the distributions of $T_{i j}^{\text {opt }}$ (resp., $R R W_{i j}\left(\Delta t_{0}\right)$ ) for each arc $i j$, perform a stochastic ranking of links according to ascending (resp., descending) $T_{i j}^{\text {opt }}$ values (see Section III-C).

\section{Stochastic Ranking}

In order to rank network links according to the distribution of their optimal repair time $T_{i j}^{\text {opt }}$ (or resilience reduction worth $R R W_{i j}\left(\Delta t_{0}\right)$ ) obtained at step 6) of the algorithm above, an approach based on the Copeland's pairwise aggregation method [37] is proposed. The Copeland's method (CM) is a simple nonparametric Condorcet method used in the political field (voting) that does not require any information about decision maker preference and operates on a multi-indicator matrix formed by $m$ objects characterized by $\Omega$ attributes [41]. CM relies on pairwise comparisons between objects in the candidate pool, and the so-called Copeland score is defined for each object as the difference between the number of times that this object beats the other objects and the number of times that it is beat by other objects.

The CM-based ranking approach applied here corresponds to a modification proposed by Al-Sharrah [42]. It first examines the $\mathrm{CDF}$ of a given variable for all the candidates, e.g., the CDF of $T_{i j}^{\mathrm{opt}}, \forall(i, j) \in E$; then, it compares the CDF of two candidates under analysis, i.e., links $i j$ and $\overline{i j}$, with respect to specific attributes $q_{k}$ of the CDF: for example, attribute $q_{k}$ may represent the $k$ th percentile. Subsequently, a quantity $S_{k}(i j, \overline{i j})$ is calculated based on a pairwise comparison between links $i j$ and $\overline{i j}$ with respect to (percentile) $q_{k}$ of the corresponding distributions $k=1, \ldots, \Omega$, as

$$
S_{k}(i j, \overline{i j})= \begin{cases}S_{k-1}(i j, \overline{i j})+1, & \text { if } q_{k}(i j) \succ q_{k}(\overline{i j}) \\ S_{k-1}(i j, \overline{i j})+0.5, & \text { if } q_{k}(i j)=q_{k}(\overline{i j}) \\ S_{k-1}(i j, \overline{i j}), & \text { if } q_{k}(i j) \prec q_{k}(\overline{i j})\end{cases}
$$

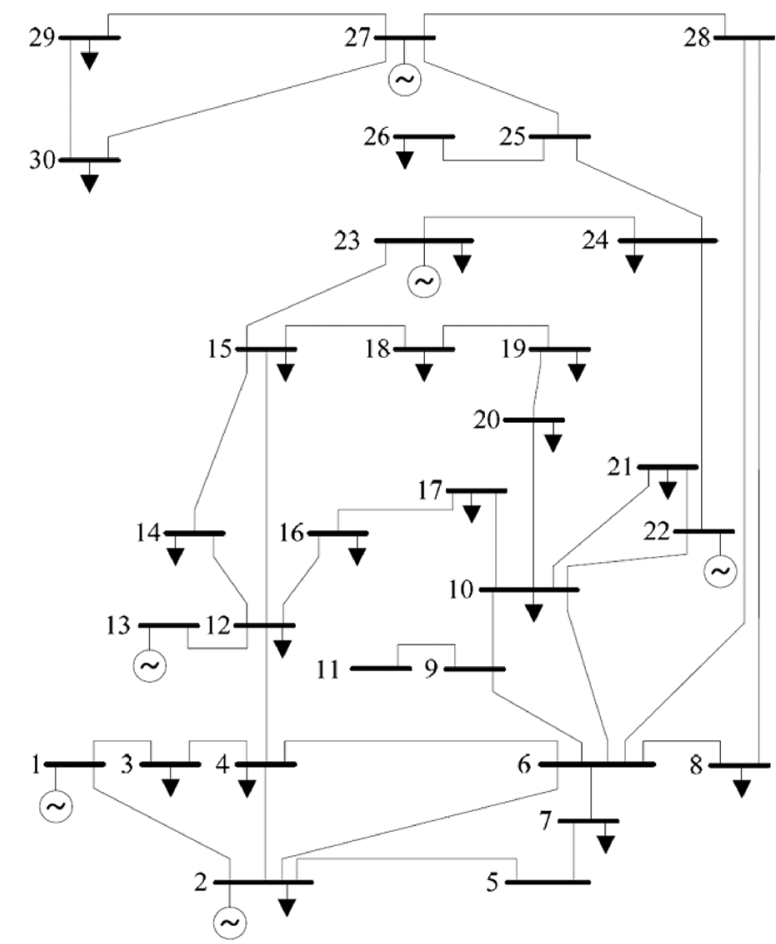

Fig. 3. Single-line diagram of the IEEE 30-bus test system.

where the sentence $q_{k}(i j) \succ q_{k}(\overline{i j})$ means that $q_{k}(i j)$ dominates $q_{k}(\overline{i j})$ with respect to the ranking rule of the variable considered, i.e., $q_{k}(i j)<q_{k}(\overline{i j})$ for $T_{i j}^{\text {opt }}$, while $q_{k}(i j)>q_{k}(\overline{i j})$ if $R R W_{i j}\left(\Delta t_{0}\right)$ is considered, while symbol " $\prec$ " represents the opposite meaning. $S_{0}(i j, \overline{i j})$ is initialized at zero for the first (percentile) $q_{1}$ and (16) is iterated through all $\Omega$ attributes (percentiles). Then, the Copeland score for each link $i j$ is defined as

$$
C(i j)=\sum_{\overline{i j} \neq i j} S_{\Omega}(i j, \overline{i j}) .
$$

This Copeland score is finally used to rank all of the links: the higher $C(i j)$, the higher the contribution of link $i j$ to the overall resilience of the network.

\section{CASE STUdy}

\section{A. Resilience-Based Criticality Measures on the IEEE 30-Bus Test System}

The IEEE 30-bus test system [43] is taken as a reference case study for the proposed resilience-based component importance measure approach. This system (Fig. 3) represents a portion of the American Electric Power System and is composed of 30 buses connected by 41 transmission lines. To carry out the analysis, each system component is transposed into a node or edge of the representative topological network, as shown in Fig. 4. Three different physical types of nodes are considered: generator nodes (where the electricity flow is fed into the network), demand nodes (where customers are connected), and transfer or transmission nodes (without customers or sources).

The simulation procedure introduced in Section III-B is then used to rank each component of the IEEE 30-bus network according to the criticality metrics introduced. In normal condi- 


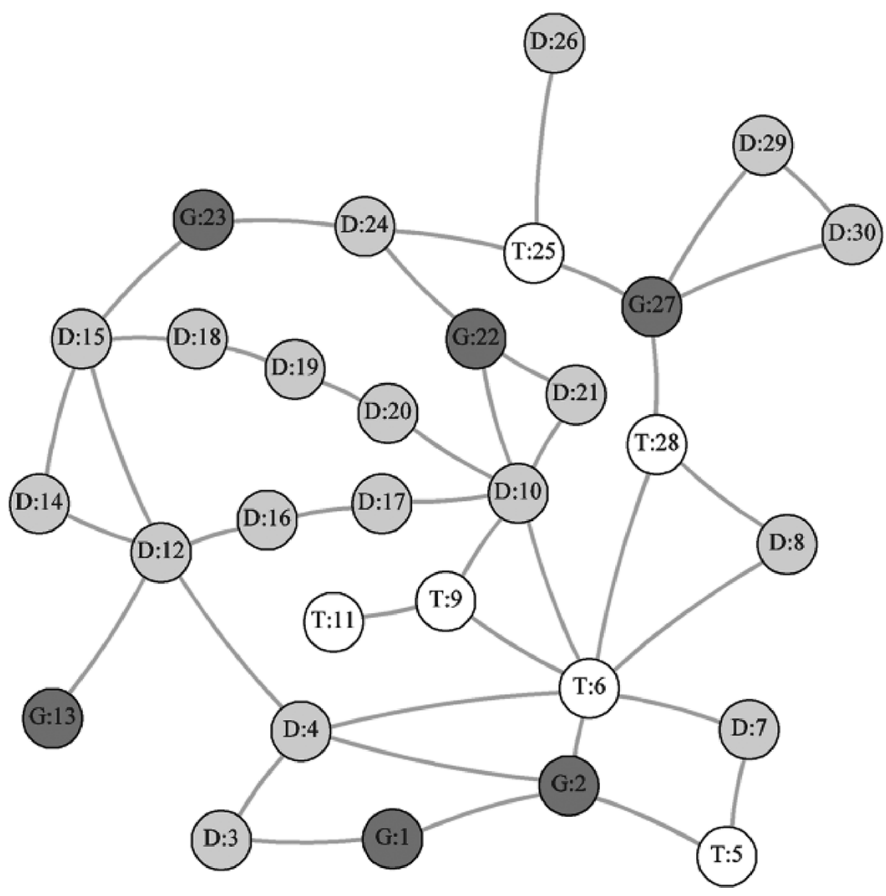

Fig. 4. Graph representation of the IEEE 30-bus test system. The dark grey circles labeled with $\mathrm{G}$ represent the generator nodes, the white circles labeled with $\mathrm{T}$ represent transmission nodes, and the light grey circles labeled with $\mathrm{D}$ represent the demand nodes.

tions, the network is assumed to operate under the following parameters setting: the generation capacity is identical for all generation nodes and equal to 60 , in arbitrary units (a.u.); the flow demands are 20 a.u. for all load nodes; the values of the transmission capacities are 20 a.u. for all of the network links. The homogeneous assignments of generation capacity, demand, and link capacity are here applied for the purpose of identifying the resilience criticalities of all of the network arcs stemming from their different topological connections. For the same reason, a constant failure probability $p_{e}(i j)$ is assumed for all of the network links under disruptive event $e$. The roulette wheel selection method [44] is used in step 2 for sampling a failure configuration by selecting a failed link at each spin until a certain number $\|E\| \cdot p_{e}(i j)=12$ of arcs are selected.

Fig. 5 illustrates the cumulative distribution functions $(\mathrm{CDFs})$ of $T_{i j}^{\text {opt }}$ for five representative links $(\langle 1,3\rangle,\langle 5,7\rangle,\langle 27,30\rangle$, $\langle 8,28\rangle$ and $\langle 10,21\rangle)$, obtained at step 5$)$ of the procedure by applying the simulation algorithm proposed in Section III-B (for $\aleph=1000$ samples). The figure illustrates the probability that $T_{i j}^{\text {opt }}$ is less than or equal to a target value $X$. It can be seen that the optimal repair time associated with link $\langle 1,3\rangle$, i.e., $T_{13}^{\text {opt }}$, will never be larger than 5 (square-line curve in Fig. 5). Moreover, the curve for link $\langle 1,3\rangle$ always "dominates" the other curves. Therefore, this link should have the highest priority to be repaired in order to maximize system resilience.

However, considering e.g., links $\langle 5,7\rangle$ (circle line) and $\langle 27,30\rangle$ (triangle line) in Fig. 5, it is not evident which one "dominates" the other, due to the intersection of their CDF curves. Thus, the CM-based ranking approach introduced in Section III-C is applied to rank the importance of the links. Fig. 6 reports the Copeland scores of all the 41 links in the

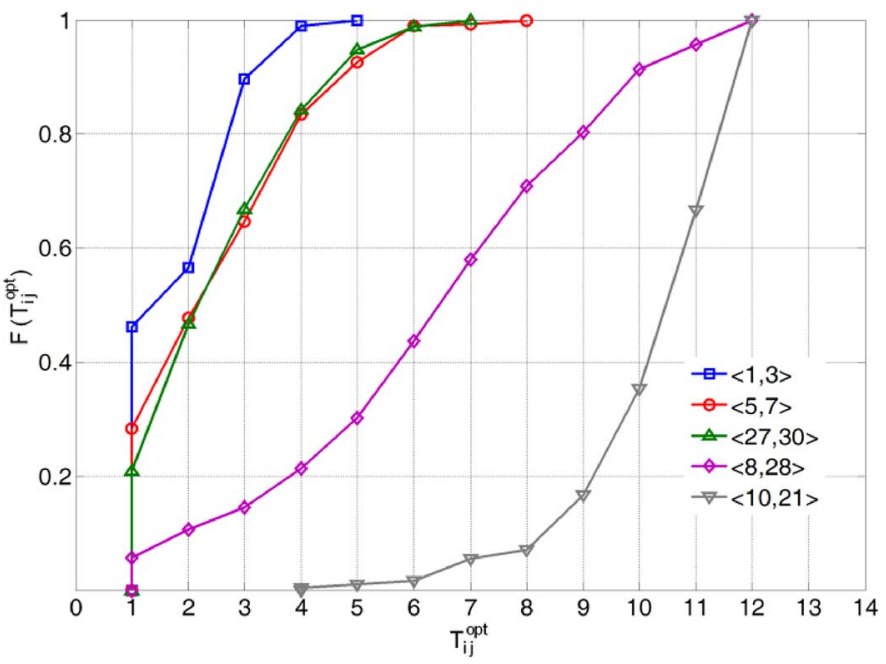

Fig. 5. Cumulative probability distributions of the optimal repair time $\mathrm{T}_{i j}^{\mathrm{opt}}$ for five representative links.

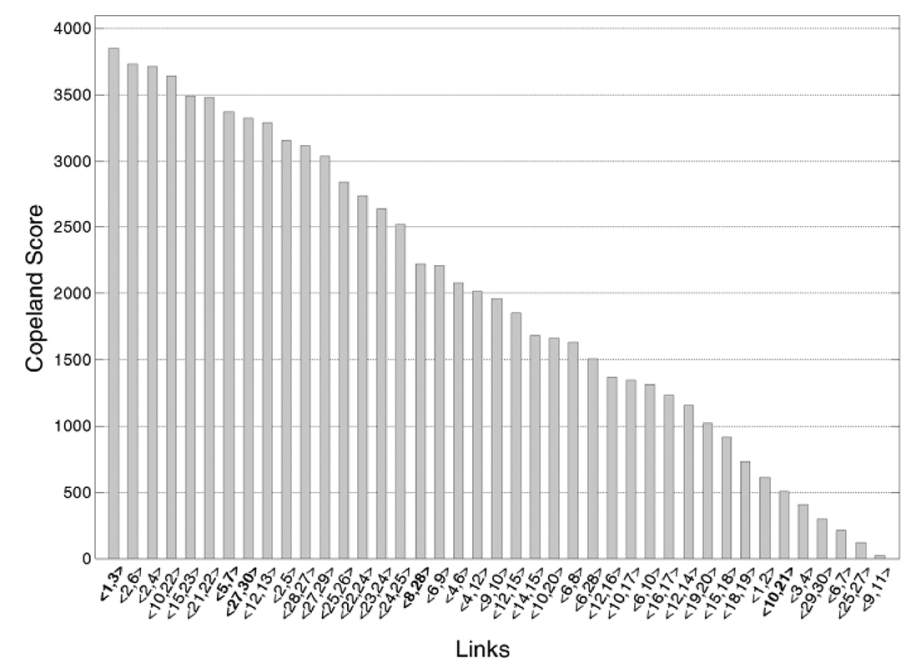

Fig. 6. Copeland score ranking of the optimal repair time $T_{i j}^{\text {opt }}$ for all IEEE 30-bus network links.

IEEE 30-bus network, ordered in descending order, with link $\langle 1,3\rangle$ having the highest score, followed by links $\langle 2,6\rangle,\langle 2,4\rangle$, $\langle 10,22\rangle$ and so forth. Furthermore, Fig. 7 graphically illustrates the Copeland score of the optimal repair time $T_{i j}^{\text {opt }}$ for all IEEE 30-bus network links, where links with higher values of Copeland score are represented as thicker and darker edges. It is shown that two types of links are more important in terms of $T_{i j}^{\mathrm{opt}}: 1$ ) the links which connect the generator nodes with the other two types of nodes (transmission nodes and demand nodes), e.g., links $\langle 2,6\rangle,\langle 1,3\rangle,\langle 12,13\rangle$, etc., and 2) the links which are the only ones connected to demand nodes, e.g., link $\langle 25,26\rangle$. The restoration of these types of links is most likely able to augment the total amount of flow received by the demand nodes of the network: thus, high priority should be given to these links when considering the repair order of the failed links.

Figs. 8 and 9 illustrate the results based on the resilience reduction worth $R R W_{i j}\left(\Delta t_{0}\right)$ for all the links and for a delay time $\Delta t_{0}=3$ units, i.e., the Copeland score ranking and its graphical representation, respectively. It is noted that different sets 


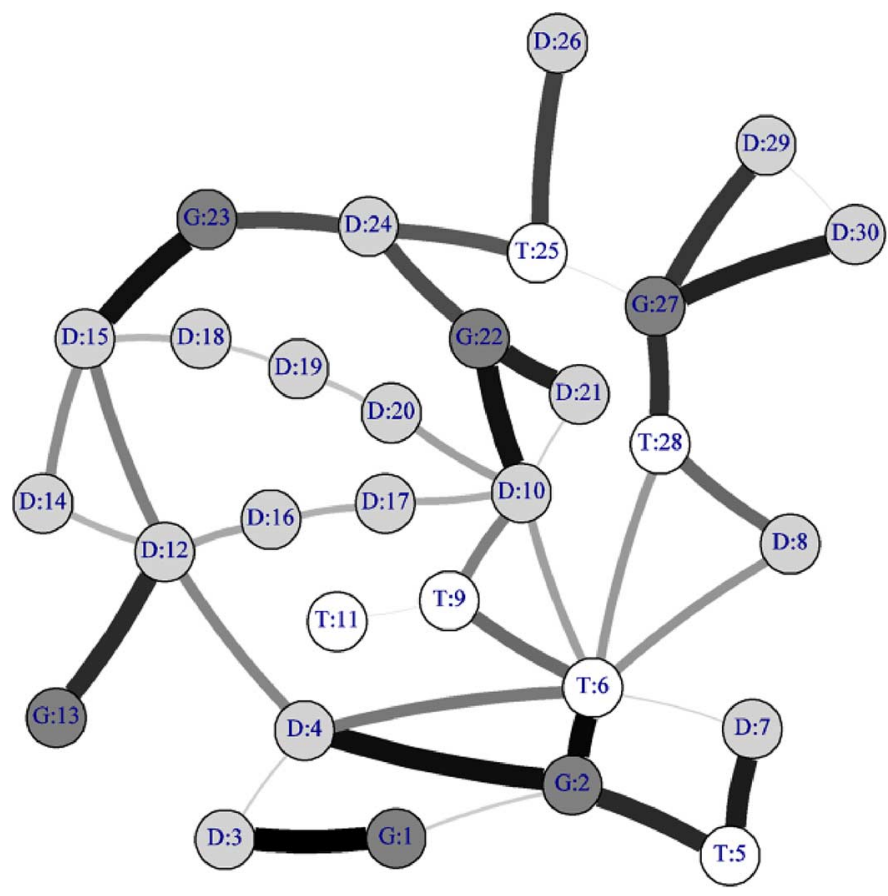

Fig. 7. Graphical illustration of the Copeland scores of the optimal repair time $T_{i j}^{\text {opt }}$ for all IEEE 30-bus network links. Links with higher value of Copeland score are represented as thicker and darker edges.

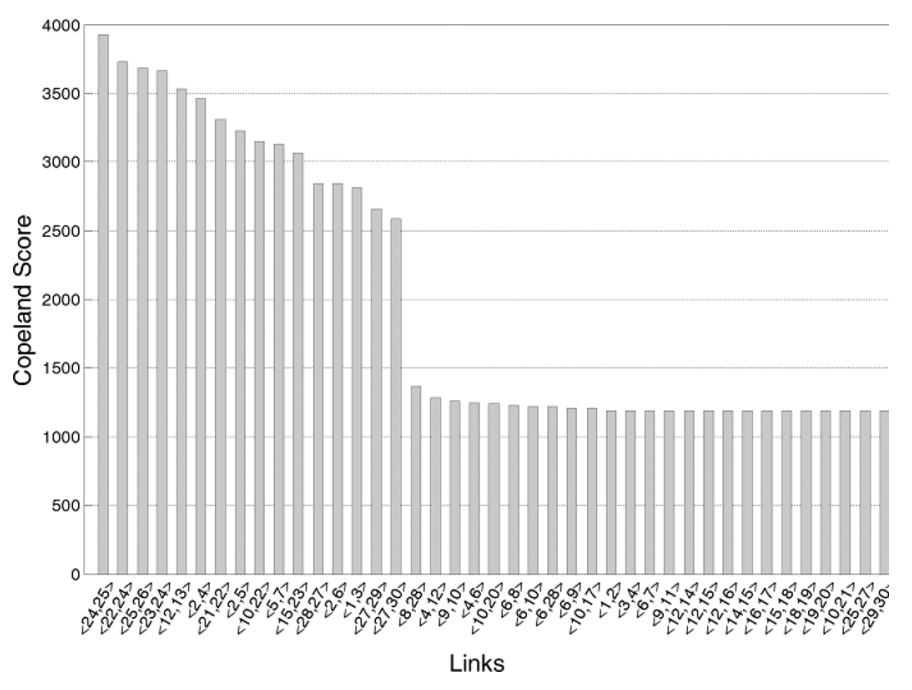

Fig. 8. Copeland score ranking of the resilience reduction worth $\operatorname{RRW}_{i j}\left(\Delta \mathrm{t}_{0}=3\right)$ for all IEEE 30-bus network links.

of values of the resilience reduction worth $R R W_{i j}\left(\Delta t_{0}\right)$ for all network links can be obtained under different values of delay $\Delta t_{0}$; however, the ranking of $R R W_{i j}\left(\Delta t_{0}\right)$ for all the links by the Copeland method will keep consistent in our study. It is shown that $\langle 24,25\rangle$ is the most critical link in terms of $R R W_{i j}$, i.e., a delay in its restoration would cause the largest reduction in system resilience among all the network links; thus, adequate resources should be given to make sure of its timely restoration. Besides, it is noted that the links with high Copeland scores in terms of the optimal repair time $T_{i j}^{\mathrm{opt}}$ also have high Copeland score ranking in terms of the resilience reduction worth $R R W_{i j}$ : the correlation coefficient between the two Copeland scores is $r\left(C_{T_{i j}^{\text {opt }}}, C_{R R W_{i j}}\right)=0.82$ for $\Delta t_{0}=3$.

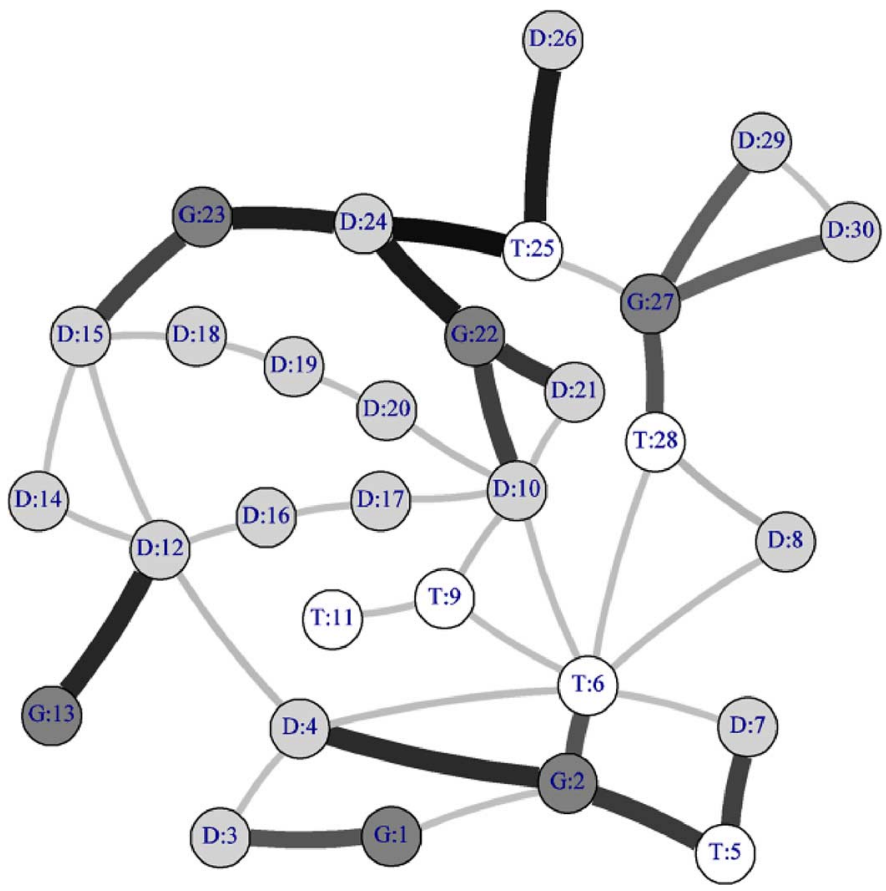

Fig. 9. Graphical illustration of the Copeland scores of the resilience reduction worth $R R W_{i j}\left(\Delta t_{0}=3\right)$ for all IEEE 30-bus network links. Links with higher values of Copeland score are represented as thicker and darker edges.

\section{B. Comparison With Betweenness Centrality Measures}

Betweenness centrality indices have been introduced as measures of component importance in a network, taking into account the different ways in which a component interacts and communicates with the rest of the network [24], [32]. A classical centrality measure is the topological betweenness centrality introduced in the social network field, which is based on the idea that a component is central if it is lies between many other components, in the sense that it is traversed by many of the shortest paths connecting pairs of nodes [24], usually called as shortest path betweenness. The topological betweenness centrality $C_{i j}^{B}$ of a given link $i j$ in a supply-demand differentiated network $G\left(V_{S} \cup V_{T} \cup V_{D}, E\right)$ is given by [38]

$$
C_{i j}^{B}=\frac{1}{\left\|V_{S}\right\| \cdot\left\|V_{D}\right\|} \sum_{s \in V_{S}, d \in V_{D}} \frac{n_{s d}(i j)}{n_{s d}}, i j \in E
$$

where $n_{s d}$ is the number of topological shortest paths between supply nodes and demand nodes, and $n_{s d}(i j)$ is the number of supply-demand shortest paths passing though link $i j$.

To account for the issue that in some cases flow may not follow the ideal geodesic paths from supply to demand nodes, a betweenness centrality measure based on the idea of maximum network flow has been proposed [28], usually known as flow betweenness. The measure counts all independent paths that carry information when a maximum flow is pumped between each pair of vertices. The flow betweenness of a component is defined as the amount of flow through it when the maximum flow is transmitted from source $s$ to demand $d$, averaged over all $s$ and $d$. It is quantitatively defined as [28]

$$
C_{i j}^{F}=\frac{\sum_{s \in V_{S}, d \in V_{D}} m_{s d}(i j)}{\sum_{s \in V_{S}, d \in V_{D}} m_{s d}}, i j \in E
$$




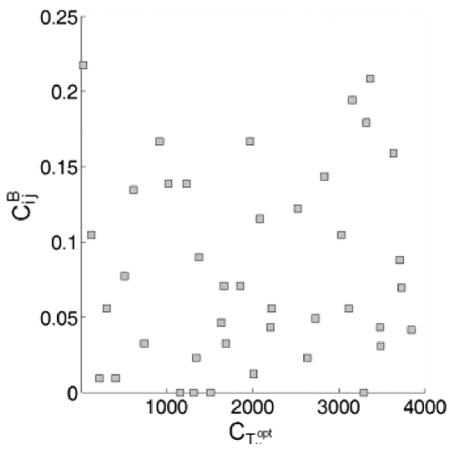

(a)

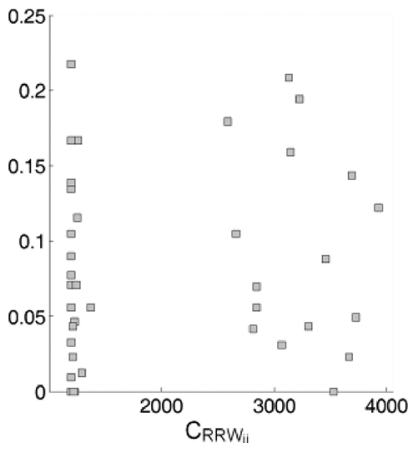

(b)

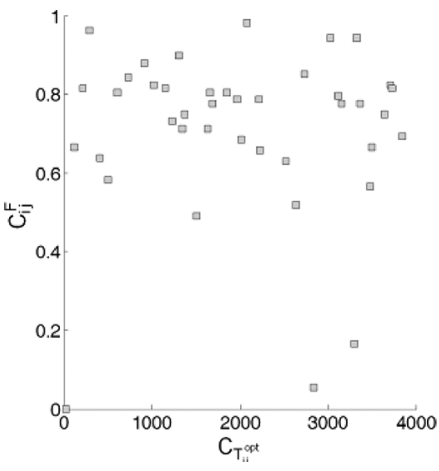

(a)

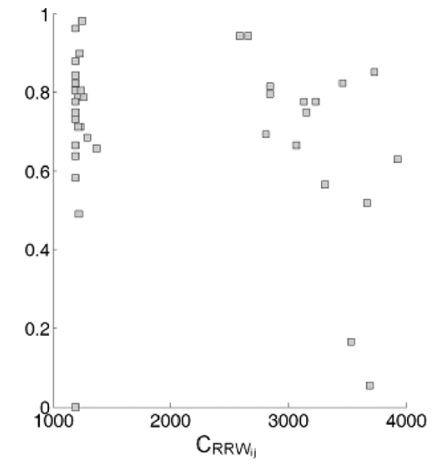

(b)

Fig. 10. Scatterplot of the Copeland scores of (a) the optimal repair time $C_{T_{i j}^{\text {opt }}}$ and (b) resilience reduction worth $C_{R R W_{i j}}$ with the shortest path betweenness $C_{i j}^{B}$ for the links of the IEEE 30-bus network.

where $m_{s d}$ is the maximum flow from a source node $s$ to a demand node $d$ and $m_{s d}(i j)$ is the maximum flow from $s$ to $d$ that passes through link $i j$.

In practical terms, however, neither of the two betweenness measures introduced above is realistic. Both count only a small subset of possible paths between vertices, and both assume some kind of optimality in information transmission (shortest paths or maximum flow). Therefore, a new betweenness measure that counts essentially all paths between vertices and which makes no assumptions of optimality has been suggested, called random walk betweenness [29]. This measure is based on random walks between vertex pairs and asks, in essence, how often a given component will fall on a random walk between another pair of vertices. Roughly speaking, the random walk betweenness of a link $i j$ is equal to the number of times that a random walk starting at $s$ and ending at $d$ passes through the link along the way, averaged over all $s$ and $d$. Let $I_{i j}^{s d}$ be the current flow from $s$ to $d$, through link $i j$. Then, the random walk betweenness of a link $i j$ is defined as

$$
C_{i j}^{R W}=\frac{1}{\left\|V_{S}\right\| \cdot\left\|V_{D}\right\|} \sum_{s \in V_{S}, d \in V_{D}} I_{i j}^{s d}, i j \in E .
$$

We are interested in comparing the ranking results of our resilience-based component importance measures to these betweenness centrality indices, i.e., shortest path betweenness, flow betweenness and random walk betweenness for the proposed IEEE 30-bus network. Fig. 10 shows the values of the Copeland scores for the optimal repair time $C_{T_{i j}^{\text {opt }}}$ (left panel) and for the resilience reduction worth $C_{R R W_{i j}}$ (right panel) plotted with respect to the shortest path betweenness $C_{i j}^{B}$ for all of the links of IEEE 30-bus network. No obvious correlation can be identified from the figures. Actually, the correlation coefficients between $C_{T_{i j}^{\text {opt }}}, C_{R R W_{i j}}$, and $C_{i j}^{B}$ are $r\left(C_{T_{i j}^{\text {opt }}}, C_{i j}^{B}\right)=0.08$ and $r\left(C_{R R W_{i j}}, C_{i j}^{B}\right)=0.1$, respectively. Similarly, Fig. 11 plots the relationship between the Copeland scores for the optimal repair time $C_{T_{i j}^{\text {opt }}}$ (left panel) and the resilience reduction worth $C_{R R W_{i j}}$ (right panel) with the flow betweenness $C_{i j}^{F}$; Fig. 12 shows the same scatterplots with respect to the random walk betweenness $C_{i j}^{R W}$. The correlation coefficients are $r\left(C_{T_{i j}^{\text {opt }}}, C_{i j}^{F}\right)=0.002, r$

Fig. 11. Scatterplot of the Copeland scores of (a) the optimal repair time $C_{T_{i j}^{\text {opt }}}$ and (b) resilience reduction worth $C_{R R W_{i j}}$ with flow betweenness $C_{i j}^{F}$ for the links of the IEEE 30-bus network.

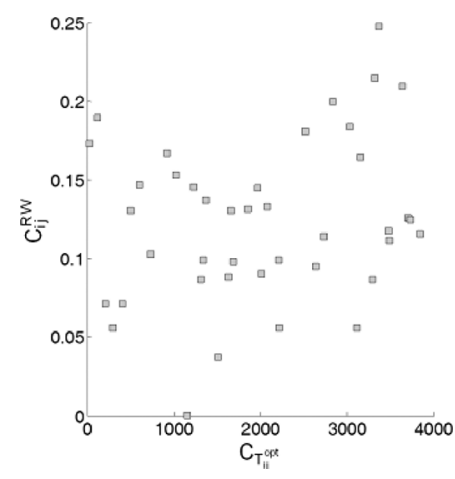

(a)

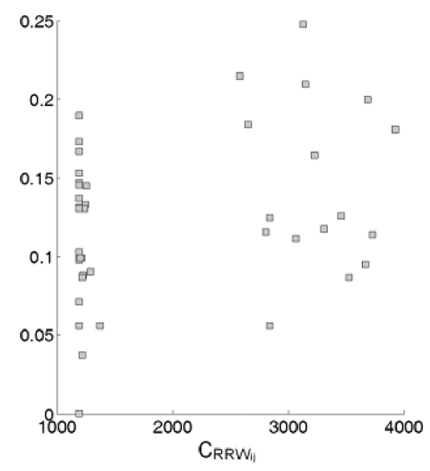

(b)
Fig. 12. Scatterplot of the Copeland scores of (a) the optimal repair time $C_{T_{i j}^{\text {opt }}}$ and (b) resilience reduction worth $C_{R R W_{i j}}$ with the random walk betweenness $C_{i j}^{R W}$ for the links of the IEEE 30-bus network.

$\left(C_{R R W_{i j}}, C_{i j}^{F}\right)=-0.24, r\left(C_{T_{i j}^{\text {opt }}}, C_{i j}^{R W}\right)=0.24$ and $r$ $\left(C_{R R W_{i j}}, C_{i j}^{R W}\right)=0.32$, respectively.

These results show that the betweenness centrality indices (e.g., shortest path betweenness, flow betweenness and random walk betweenness) do not capture the component criticality with respect to resilience for the recovery of the IEEE 30-bus network. This implies that these centrality measures (which are calculated under normal operation condition) are not applicable to guide the system restoration after a disruptive event, e.g., to prepare an efficient component repair priority checklist in the event of system failure.

\section{CONCLUSION}

This paper primarily contributes two metrics to measure the criticality of network components from the perspective of their contribution to system resilience, defined as the cumulative system functionality that has been restored at time $t$, normalized by the expected cumulative system functionality supposing that the system has not been affected by disruption during this time period.

The first resilience-based component importance measure, i.e., the optimal repair time $T_{i j}^{\mathrm{opt}}$ in (14), offers an explicit quantification of the priority that should be given to arc $i j$ to be repaired and re-installed into the network. Lower values of $T_{i j}^{\mathrm{opt}}$ 
indicate higher priority, i.e., higher rank in the component repair checklist for system restoration in the event of system failure. The second resilience-based component importance measure, i.e., the resilience reduction worth $R R W_{i j}\left(\Delta t_{0}\right)$, quantifies the potential loss in optimal system resilience due to a delay $\Delta t_{0}$ in the repair time of link $i j$. This measure can provide valuable information to guide the recovery process of a particular component: components with high values of $R R W_{i j}\left(\Delta t_{0}\right)$ should be given high priority to their timely restoration, e.g., be assigned with adequate restoration resources.

Given the stochastic nature of disruptive events on an infrastructure network, a Monte Carlo-based method has been proposed to generate distributions of optimal repair time $T_{i j}^{\mathrm{opt}}$ and resilience reduction worth $R R W_{i j}\left(\Delta t_{0}\right)$ for all the components in the network; then, a stochastic ranking approach based on the Copeland's pairwise aggregation method has been applied to rank components importance.

The results of the two measures applied to the IEEE 30-bus test network demonstrate some non-obvious and meaningful conclusions about the contributions of certain links to the resilience of the network. It is shown that two types of links are most important in terms of $\left.T_{i j}^{\mathrm{opt}}: 1\right)$ the links which connect generator nodes with the other two types of nodes (transmission nodes and demand nodes), e.g., links $\langle 2,6\rangle,\langle 1,3\rangle,\langle 12,13\rangle$ etc., and 2) the link which is the only arc connecting to demand nodes, i.e., link $\langle 25,26\rangle$. The restoration of these types of links is most likely able to augment the total amount of flow received by the demand nodes of the network so that high priority should be given to these links in the reparation list. Besides, those links with high Copeland scores in terms of $T_{i j}^{\text {opt }}$ also have high Copeland scores ranking in terms of the resilience reduction worth $R R W_{i j}$ : actually, the correlation coefficient between the two quantities is $r\left(C_{T_{i j}^{\text {opt }}}, C_{R R W_{i j}(3)}\right)=0.82$.

It is noted that the differences of the resilience-based CIMs proposed in the present paper with respect to that in [35] mainly fall into the following two aspects: 1) the concept and definition of system resilience which the CIMs rely on is an non-memoryless one in this study by taking into account the cumulative restored system functionality and 2) the focus of the proposed resilience-based CIMs is to quantify the effect that the recovery, rather than the disruption, of individual components has on the global system resilience, thus, are valuable in suggesting the most effective way for system (recovery) operation.

Finally, it is shown that the classical betweenness centrality indices, such as the shortest path betweenness, flow betweenness and random walk betweenness, do not capture resilience criticality as do the resilience-based measures $T_{i j}^{\mathrm{opt}}$ and $R R W_{i j}\left(\Delta t_{0}\right)$. Actually, the existing centrality indices and the CIMs in the reliability engineering literature are not appropriate to help implement resilience planning because they do not take into account system recovery time. Instead, the two measures proposed in the present paper provide insights useful for practical restoration activities of infrastructure networks after suffering a disruptive event.

It is noted that in this study we consider a system suffering a specific type of events e (e.g., earthquakes of a certain magnitude) and rank the component importance by a stochastic ranking method. The results are valuable to help implement the system restoration if this type of event happens on the system. However, it might be possible that an infrastructure system is affected by different types of events concurrently (e.g., earthquake and tsunami). Future studies will concentrate on the application of the resilience-based component importance measures to different types of infrastructure networks subject to (possibly different types of correlated) disruptive events, in order to further demonstrate the practical effectiveness of the measures.

\section{ACKNOWLEDGMENT}

The authors would like to thank the anonymous referees for providing helpful comments and suggestions for our paper.

\section{REFERENCES}

[1] E. Zio, The Monte Carlo Simulation Method for System Reliability and Risk Analysis. London, U.K.: Springer, 2013, p. 198p.

[2] T. G. Lewis, Critical Infrastructure Protection in Homeland Security: Defending a Networked Nation. Hoboken, NJ, USA: Wiley, 2006.

[3] W. Clinton, "Presidential Decision Directive PDD-63, Protecting America's Critical Infrastructures," Washington, DC, USA, 1998.

[4] G. W. Bush, "Homeland Security Presidential Directive-3 (HSPD-3)," Washington, DC, USA, 2002.

[5] G. W. Bush, "Homeland Security Presidential Directive-7 (HSPD-7)," Washington, DC, USA, 2003.

[6] C. Pursiainen, "The challenges for European critical infrastructure protection," Eur. Integration, vol. 31, no. 6, pp. 721-739, 2009.

[7] B. Obama, "Presidential Policy Directive 21: Critical Infrastructure Security and Resilience," Washington, DC, USA, 2013.

[8] G. P. Cimellaro, A. M. Reinhorn, and M. Bruneau, "Framework for analytical quantification of disaster resilience," Eng. Structures, vol. 32, no. 11, pp. 3639-3649, 2010.

[9] J. D. Moteff, "Critical Infrastructure Resilience: The Evolution of Policy and Programs and Issues for Congress," Congressional Res. Service, 2012

[10] E. Zio, "Reliability engineering: Old problems and new challenges," Rel. Eng. Syst. Safety, vol. 94, no. 2, pp. 125-141, 2009.

[11] M. Ouyang, "Review on modeling and simulation of interdependent critical infrastructure systems," Rel. Eng. Syst. Safety, vol. 121, pp. 43-60, 2014.

[12] Z. W. Birnbaum, "On the importance of different components in a multicomponent system," in Multivariate Analysis. New York, NY, USA: Academic, 1969, vol. 2.

[13] J. Fussell, "How to calculate system reliability and safety characteristics," IEEE Trans. Rel., vol. TEL-24, no. 3, pp. 169-174, Aug. 1975.

[14] A. Gandini, "Importance and sensitivity analysis in assessing system reliability," IEEE Trans. Rel., vol. 39, no. 1, pp. 61-70, Apr. 1990.

[15] G. Levitin, L. Podofillini, and E. Zio, "Generalized importance measures for multistate elements based on performance level restrictions," Rel. Eng. Syst. Safety, vol. 82, pp. 63-73, 2003.

[16] J. E. Ramirez-Marquez and D. W. Coit, "Composite importance measures for multi-state systems with multi-state components," IEEE Trans. Rel., vol. 54, no. 3, pp. 517-529, Sep. 2005.

[17] J. E. Ramirez-Marquez and D. W. Coit, "Multi-state component criticality analysis for reliability improvement in multi-state systems," Rel. Eng. Syst. Safety, vol. 92, no. 12, pp. 1608-1619, 2007.

[18] J. D. Andrews and S. Beeson, "Birnbaum's measure of component importance for noncoherent systems," IEEE Trans. Rel., vol. 52, no. 2, pp. 213-219, Mar. 2003.

[19] W. Wang, J. Loman, and P. Vassiliou, "Reliability importance of components in a complex system," in Proc. IEEE Annu. Symp. Rel. Maintainability, 2004, pp. 6-11.

[20] Y.-P. Fang and E. Zio, "Hierarchical modeling by recursive unsupervised spectral clustering and network extended importance measures to analyze the reliability characteristics of complex network systems," Amer. J. Oper. Res., vol. 3, no. 1A, pp. 101-112, 2013.

[21] S. P. Borgatti, "Centrality and network flow," Social Networks, vol. 27, no. 1, pp. 55-71, 2005. 
[22] W. Kröger and E. Zio, Vulnerable Systems. Berlin, Germany: Springer, 2011.

[23] J. Nieminen, "On the centrality in a graph," Scandinavian J. Psychol., vol. 15 , no. 1 , pp. 332-336, 1974.

[24] L. C. Freeman, "Centrality in social networks conceptual clarification," Social Networks, vol. 1, no. 3, pp. 215-239, 1979.

[25] G. Sabidussi, "The centrality index of a graph," Psychometrika, vol. 31, no. 4, pp. 581-603, 1966.

[26] S. Wasserman and K. Faust, Social Network Analysis: Methods and. Applications. Cambridge, U.K., ENG: Cambridge Univ., 1994.

[27] V. Latora and M. Marchiori, "A measure of centrality based on network efficiency," New J. Phys., vol. 9, no. 6, p. 188, 2007.

[28] L. C. Freeman, S. P. Borgatti, and D. R. White, "Centrality in valued graphs: A measure of betweenness based on network flow," Social Networks, vol. 13, no. 2, pp. 141-154, 1991.

[29] M. E. Newman, "A measure of betweenness centrality based on random walks," Social Networks, vol. 27, no. 1, pp. 39-54, 2005.

[30] E. Jenelius, T. Petersen, and L. G. Mattsson, "Importance and exposure in road network vulnerability analysis," Transportation Res. A: Policy and Practice, vol. 40, no. 7, pp. 537-560, 2006.

[31] P. Hines and S. Blumsack, "A centrality measure for electrical networks," in Proc. IEEE 41st Annu. Hawaii Int. Conf. Syst. Sci., Jan. 2008, pp. 185-185.

[32] E. Zio and R. Piccinelli, "Randomized flow model and centrality measure for electrical power transmission network analysis," Rel. Eng. Syst. , vol. 95, no. 4, pp. 379-385, 2010.

[33] E. Zio and G. Sansavini, "Component criticality in failure cascade processes of network systems," Risk Anal., vol. 31, no. 8, pp. 1196-1210, 2011.

[34] B. Natvig, A. B. Huseby, and M. O. Reistadbakk, "Measures of component importance in repairable multistate systems-A numerical study," Rel. Eng. Syst. Safety, vol. 96, no. 12, pp. 1680-1690, 2011.

[35] K. Barker, J. E. Ramirez-Marquez, and C. M. Rocco, "Resilience-based network component importance measures," Rel. Eng. Syst. Safety, vol. 117, pp. 89-97, 2013.

[36] H. Baroud, K. Barker, and J. E. Ramirez-Marquez, "Importance measures for inland waterway network resilience," Transportation Res. E: Logistics and Transportation Review, vol. 62, pp. 55-67, 2014.

[37] V. R. Merlin and D. G. Saari, "Copeland method II: Manipulation, monotonicity, and paradoxes," J. Economic Theory, vol. 72, no. 1, pp. 148-172, 1997.

[38] R. Kinney, P. Crucitti, R. Albert, and V. Latora, "Modeling cascading failures in the North American power grid," Eur. Phys. J. B, Conden. Matter and Complex Syst., vol. 46, no. 1, pp. 101-107, 2005.

[39] Cplex Optimization Studio IBM ILOG, Aug. 2014 [Online]. Available: http://www-03.ibm.com/software/products/en/ibmilogcpleoptistud

[40] J. F. Espiritu, D. W. Coit, and U. Prakash, "Component criticality importance measures for the power industry," Electr. Power Syst. Res., vol. 77, no. 5, pp. 407-420, 2007.

[41] J. C. Pomerol and S. Barba-Romero, Multicriterion Decision in Management: Principles and Practice. Berlin, Germany: Springer, 2000, vol. 25.

[42] G. Al-Sharrah, "Ranking using the Copeland score: A comparison with the Hasse diagram," J. Chem. Inf. Modeling, vol. 50, no. 5, pp. 785-791, 2010.

[43] Power System Test Case Archive, , Sep. 2014 [Online]. Available: http:/ /www.ee.washington.edu/research/pstca/

[44] A. Lipowski and D. Lipowska, "Roulette-wheel selection via stochastic acceptance," Physica A: Statistical Mechanics and its Applications, vol. 391, no. 6, pp. 2193-2196, 2012.

[45] R. E. Barlow and F. Proschan, "Importance of system components and fault tree events," Stoch. Process Appl., vol. 3, pp. 153-73, 1975.

[46] B. Natvig, "New light on measures of importance of system components," Scand. J. Stat., vol. 12, pp. 43-54, 1985.

[47] B. Natvig, Multistate Systems Reliability Theory with Applications. Hoboken, NJ, USA: Wiley, 2011.

[48] B. Natvig, "Measures of component importance in nonrepairable and repairable multistate strongly coherent systems," Methodology and Computing in Appl. Probabil., vol. 13, no. 3, pp. 523-547, 2011.

[49] Y.-P. Fang, "Critical infrastructure protection by advanced modelling, simulation and optimization for cascading failure mitigation and resilience," Ph.D. dissertation, Ind. Eng. Dept., Ecole Centrale Paris, Paris, France, 2015.

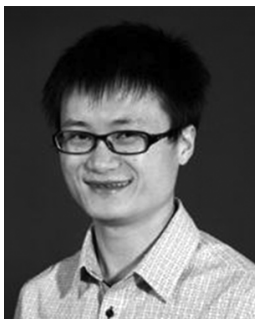

Yi-Ping Fang received the B.S. and M.S. degrees in electronic and information engineering from Beihang University, Beijing, China, in 2009 and 2012, respectively, and the Ph.D. degree in industrial engineering within the Chair on Systems Science and the Energetic Challenge, European Foundation for New Energy - Électricité de France, École Centrale Paris, France.

$\mathrm{He}$ is currently a Postdoctoral Fellow with the Laboratory of Risk and Reliability, Department of Mechanical and Process Engineering, ETH Zurich, Zurich, Switzerland. His research interest focuses on the study of advanced modelling, simulation, and optimization methods for the risk, vulnerability, and resilience analysis of complex networked engineering systems.

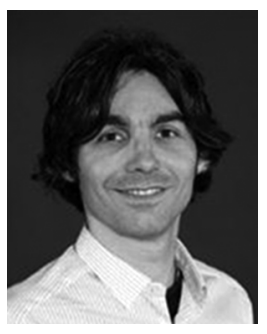

Nicola Pedroni received the B.S. degree in energetic engineering, M.Sc. degree in nuclear engineering, and $\mathrm{Ph} . \mathrm{D}$. degree in radiation science and technology from Politecnico di Milano, Milan, in 2003, 2005, and 2010, respectively.

$\mathrm{He}$ is an Assistant Professor with the Electricité de France (EDF) Chair on System Sciences and Energetic Challenge with a joint appointment at the Ecole Centrale Pairs (ECP) (Chatenay-Malabry, France) and Ecole Superieure d'Electricite (SUPELEC) (Gif-Sur-Yvette, France). He has been an Assistant Professor with Nuclear Power Plants at the Politecnico di Milano (Milano, Italy). During his Ph.D., he visited the Department of Nuclear Science and Engineering of the MIT (2008-2009). He is a coauthor of approximately 20 papers on international journals and four chapters in international books. His research focuses on the study of computational methods for the risk analysis of safety critical systems, e.g., Monte Carlo methods for reliability estimation, theories for uncertainty representation, soft-computing techniques for regression modeling, and algorithms for solving optimization problems.

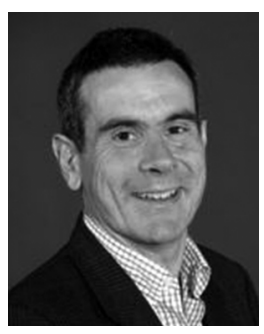

Enrico Zio (M'06-SM'09) received the B.S. degree in nuclear engineering from Politecnico di Milano, Milan, Italy, in 1991, the M.Sc. degree in mechanical engineering from the University of California, Los Angeles, CA, USA, in 1995, the Ph.D. degree in nuclear engineering from the Politecnico di Milano in 1995, and the Ph.D. degree in nuclear engineering from the Massachusetts Institute of Technology, Cambridge, MA, USA, in 1998.

$\mathrm{He}$ is the Director of the Chair on Systems Science and the Energetic Challenge of the European Foundation for New Energy of Électricité de France (EDF) at Ecole Centrale Paris and Supelec, a Full Professor, President and Rector's delegate of the Alumni Association and past-Director of the Graduate School at Politecnico di Milano, and Adjunct Professor with University of Stavanger. His research focuses on the characterization and modeling of the failure/repair/maintenance behavior of components, complex systems and critical infrastructures for the study of their reliability, availability, maintainability, prognostics, safety, vulnerability and security, mostly using a computational approach based on advanced Monte Carlo simulation methods, soft computing techniques and optimization heuristics. $\mathrm{He}$ is author or coauthor of five international books and more than 170 papers on international journals.

Prof. Zio is the Chairman of the European Safety and Reliability Association ESRA, member of the scientific committee of the Accidental Risks Department of the French National Institute for Industrial Environment and Risks, member of the Korean Nuclear society and China Prognostics and Health Management society, and past-Chairman of the Italian Chapter of the IEEE Reliability Society. He is serving as an associate editor of the IEEE TRANSACTIONS ON RELIABILITY and as editorial board member in various international scientific journals, among which Reliability Engineering and System Safety, Journal of Risk and Reliability, International Journal of Performability Engineering, Environment, Systems and Engineering, and International Journal of Computational Intelligence Systems. He has functioned as Scientific Chairman of three International Conferences and as Associate General Chairman of two others. 